\title{
Difficult medical encounters and job satisfaction - results of a cross sectional study with general practitioners in Germany
}

Katja Goetz ${ }^{*}$ (D) Janis Mahnkopf, Anna Kornitzky and Jost Steinhäuser

\begin{abstract}
Background: In primary care 15\% of patient encounters are perceived as challenging by general practitioners (GP). However it is unknown what impact these encounters have regarding job satisfaction. The aim of this study was to evaluate which encounters are perceived as challenging by German GPs and whether they were associated with job satisfaction.

Methods: A total of 1538 questionnaires were sent to GPs in the federal state of Schleswig-Holstein, Germany. GPs should rate 14 medical conditions and 8 traits of patients on the perceived challenge using a Likert scale (1: 'not challenging at all' to 10: 'extremely challenging'). Job satisfaction was measured with the Warr-Cook-Wall job satisfaction scale. A linear regression analyses were used to explore potential associations between for the primary outcome variable 'overall job satisfaction'.

Results: Total response was 578 (38\%). GPs perceived 16\% of their patients as challenging. Psychiatric disorders such as somatization disorder (mean $=7.42)$, schizophrenia (mean $=6.83)$ and anxiety disorder $($ mean $=6.57)$ were ranked as high challenging while diabetes mellitus type $2($ mean $=4.87)$ and high blood pressure $($ mean $=3.22)$ were ranked as a rather low challenging condition. GPs were mostly satisfied with 'colleagues' (mean $=5.80)$ and mostly dissatisfied with their 'hours of work' (mean $=4.20$ ). The linear regression analysis showed no association with challenging medical conditions and traits of patients but only with different aspects of job satisfaction concerning the outcome variable 'overall job satisfaction'.

Conclusions: Especially psychiatric conditions are perceived as challenging the question arises, in what amount psychiatric competences are gained during the postgraduate specialty training in general practice and if GPs with a mandatory rotation in psychiatry perceive these conditions as less challenging. Interestingly this study indicates that challenging encounter in terms of challenging medical conditions and traits of patients do not affect GP's job satisfaction.
\end{abstract}

Keywords: Cross-sectional study, Difficult encounter, General practitioner, Job satisfaction, Primary health care, Psychiatric condition

\footnotetext{
*Correspondence: katja.goetz@uni-luebeck.de

Institute of Family Medicine, University Hospital Schleswig-Holstein, Campus

Luebeck, Ratzeburger Allee 160, 23538 Luebeck, Germany
}

(c) The Author(s). 2018 Open Access This article is distributed under the terms of the Creative Commons Attribution 4.0 International License (http://creativecommons.org/licenses/by/4.0/), which permits unrestricted use, distribution, and reproduction in any medium, provided you give appropriate credit to the original author(s) and the source, provide a link to the Creative Commons license, and indicate if changes were made. The Creative Commons Public Domain Dedication waiver (http://creativecommons.org/publicdomain/zero/1.0/) applies to the data made available in this article, unless otherwise stated. 


\section{Background}

There is some evidence, that General Practitioners (GP) might perceive $15 \%$ of their patients as "difficult" which mostly include psychiatric disorders such as somatoform disorder, panic disorder, generalized anxiety and depressive disorder [1, 2]. Common medical disorders such as hypertension, diabetes, cardiac disease, or cancer were not perceived as difficult from the perspective of GPs $[1,2]$. The occurring difficulties are based on factors that are due $>$ to the patient, the physician, the situation or a combination of these factors [3, 4]. Because of this multidirectional relationship, the term 'difficult encounter' rather than 'difficult patient' is suggested [5]. Difficult encounters are capable of frustrating the patient and physician, for which both are responsible $[3,4]$. When focusing on the patient's factors, besides their medical condition, their personality and way of dealing with their condition can be a challenge for the GP [6]. GPs difficulties dealing with challenging encounters might also be based on a lack of empathy and understanding, when it comes to doctor-patient communication [3]. Therefore, communication models to improve communication skills for challenging encounters were developed [3, 4]. Challenging encounters can also be caused by missing expertise concerning a specific medical condition and the feeling to be compromised as a healer in such a situation [3].

GPs with a great amount of challenging encounters report lower job satisfaction [7]. Reasons for this are multiple. For example, challenging patients are often described as 'high utilizers' of the health care system and, by that increase workload for the GP [4]. High workload affects job satisfaction [8]. This endanger physician's health: Physicians with high workload and low job satisfaction are more likely to go on to experience substance abuse, burnout, depression or death $[7,9]$. Another finding is that low job satisfaction and low physician's wellness lead to poorer quality of patient care [9]. Thus job satisfaction could be an important factor and acts as an indicator for the quality of care.

The aim of this study was to evaluate which encounters are perceived as challenging by German GPs and whether they were associated with job satisfaction.

\section{Methods}

\section{Design and participants}

The study was confirmed to the STROBE-Guidelines (Strengthening the Reporting of Observational Studies in Epidemiology) [10]. This cross sectional study was conducted in Schleswig-Holstein, a federal state in northern Germany. A total of 1538 questionnaires were sent to all GPs of this federal state by mail. In Germany the expression GP includes general internists and general practitioners. Therefore, there was no differentiation between these groups in the questionnaire. As an alternative for completing the questionnaire there was a short-responsesheet which could be completed and returned instead. The addresses of the GPs' surgeries were obtained from the website 'https://arztsuche.kvsh.de/' of the regional Association of Statutory Health Insurance Physicians. No reminder was sent out. The survey was conducted between June and July 2015. The return of the anonymous paperbased questionnaire was classified as informed consent. Because this was an exploratory study, no power calculation was determined.

\section{Measures}

Personal and practice characteristics were measured in the questionnaire including gender, age, duration of employment in the practice and the location of the practice (measured by the car registration number of their administrative district). The duration of employment as GP was grouped in four groups: 'less than 5 years,' 5 to 10 years,' '11 to 20 years' or 'more than 20 years'. Moreover, the location of the practice measured by the car registration number was grouped into urban, medium-size town and rural area on the basis of the Federal Institute for Research on Building, Urban Affairs and Spatial Development (BBSR) [11].

Job satisfaction was measured with the German modified version of the Warr-Cook-Wall job satisfaction scale developed by Warr et al. [12, 13]. It is a well-known instrument which was validated in a large cohort of Australian medical practitioners [13]. This instrument consists of 10 items, overall job satisfaction (1 item) and 9 items to different aspects of satisfaction with work (amount of variety in job, opportunity to use abilities, freedom of working method, amount of responsibility, physical working condition, hours of work, income, recognition for work, and colleagues and fellow workers). Each item is rated on a 7-point Likert scale $(1=$ extreme dissatisfied to $7=$ extreme satisfied). A higher overall mean score indicates higher job satisfaction. Cronbach's $\alpha$ of the job satisfaction scale in this study was 0.607.

GPs were asked to estimate the percentage of challenging patients in their practice. Difficult medical encounters were operationalized with respect to two subjects: challenging medical conditions and challenging traits of patients. For the measurement of how challenging GPs perceive different medical conditions and different traits of patients, a questionnaire was developed consisting of 14 medical conditions and 8 traits. The conditions and traits chosen were defined by a selective literature search $[3,4,6,7]$. GPs were asked to assess the challenges they perceive regarding these medical conditions and traits on a 10-point Likert scale. They could choose between ' $1=$ not challenging at all' and ' $10=$ very challenging'. A high mean score indicates high challenge for the specific medical condition and the specific traits. Cronbach's $\alpha$ of 
the measurement of different medical conditions was 0.858 and of different traits of patients was 0.739 . The questionnaire of this survey was added as Additional file 1.

As an alternative for non-participation, a short-responsesheet was offered which evaluated only sociodemographic data like age and gender and reasons for non-participation.

\section{Data analysis}

Analyses were performed using SPSS 24.0 (SPSS Inc., IBM). Continuous data was summarized using means and standard deviations. Categorical data was presented as frequency counts and percentage. Moreover, means, standard deviations and $95 \%$ confidence intervals of job satisfaction scale, challenging medical conditions and challenging traits of patients were reported. Pearson's correlation was used to find out which the independent variables individual characteristics, aspect of job satisfaction, challenging medical conditions and challenging traits of patients showed a significant correlation with the dependent variable 'overall job satisfaction'. Afterwards, a linear regression analyses were used to explore potential associations between the dependent variable 'overall job satisfaction' and independent variables which correlated significantly with the dependent variable. Additionally, the possibility for multicollinearity was considered. The variance inflation factor (VIF) and the value of tolerance were reported for the last step of both regression models. Values for VIF should not be over 5.0 and for tolerance not lower than 0.25 [14]. An alpha level of $P<0.05$ was used for tests of statistical significance.

\section{Ethical approval}

Ethical approval for this research study was obtained from the University of Luebeck in May 2015 (Approval No. 15-110). No additional data were evaluated.

\section{Results}

Total response was 578 (38\%). Out of these 470 returned the questionnaire (31\%) and 108 returned the short-response-sheet (7\%). A flowchart of the study sample is presented in Fig. 1. The demographic data of those participants returning the questionnaire and the short-response-sheet is shown in Table 1. These differed significantly concerning age and gender. The mean age of the participants returning the questionnaire was $55.0(\mathrm{SD}=7.9)$ and of the short-response-sheet was 58.0 (SD 9.0). Over 34\% of participants were women, whereas of the short-response-sheet $50 \%$ were women. Most physicians were practicing for more than 20 years (43\%) or between 11 and 20 years (29\%). Young professionals, defined as practicing for less than 5 years, were the smallest group (7\%). More than half of the participants were located in medium-size towns. The others were located in urban $(25 \%)$ or rural $(18 \%)$ areas. GPs perceived
Database included $n=1,538$ General Practitioners (GP). They received a full questionnaire and short-response sheet

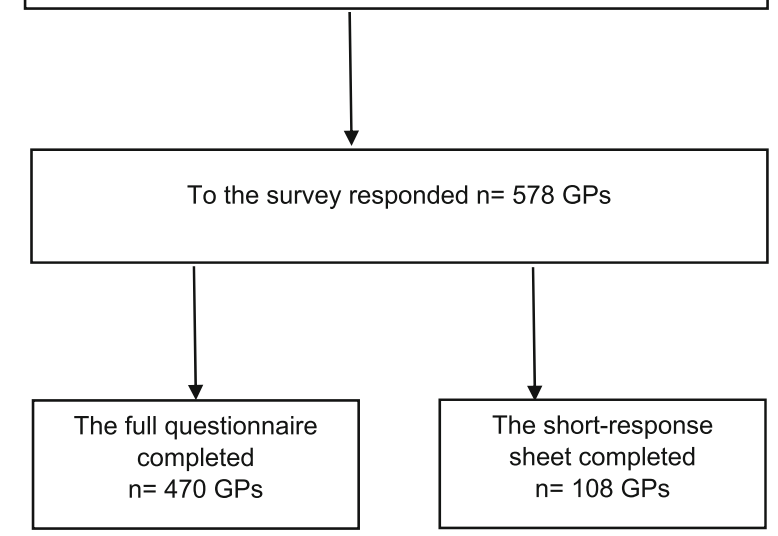

Fig. 1 Flowchart of the study sample

$16 \%$ of their patients as challenging. Younger GPs perceived a higher rate of patients as challenging.

Most of the short-response-sheet -participants $(n=53$, 49.0\%) claimed that they do not work as GPs but in a different specialty or already retired. Of the others $22 \%$ $(n=24)$ felt, that they had no time to complete the questionnaire. Another 14\% $(n=15)$ stated that they do not participate in surveys in general.

Table 2 shows the mean score of the different items measuring job satisfaction. GPs were mostly satisfied with 'colleagues and fellow workers' $($ mean $=5.80)$. They were mostly dissatisfied with their 'hours of work' $($ mean $=4.20)$, 'income' $($ mean $=4.47)$ and 'physical working condition' (mean $=4.91)$.

Table 3 shows the mean score of different medical conditions which were perceived as challenging. Somatization disorder, chronic pain and addiction to alcohol were ranked top three of challenging conditions with a mean score of more than seven points. Diabetes type 2, failure and high blood pressure had the lowest mean score on the scale (below 5 points).

Different challenging patient traits are presented in Table 4. Patients who experienced as aggressive and demanding were ranked high as challenging with a mean of 7.88 respectively 7.85 . In contrast patients who experienced as critical and anxious were ranked low as challenging with a mean of 4.87 respectively 4.37 .

The results of the correlation showed that the demographic data 'age,' 'gender' and 'employment as GP' correlated strong with the dependent variable "overall job satisfaction". The different aspects of job satisfaction showed a strong correlation to the dependent variable.

For the medical conditions 'heart failure,' 'diabetes type 2,' 'chronic renal failure', and 'anxiety disorder' and for the patient traits 'anxious', 'unfriendly', 'obsessive-compulsive 
Table 1 Sociodemographic data of participants and short-response-sheet -participants $(n=577)$

\begin{tabular}{|c|c|c|c|c|}
\hline \multicolumn{2}{|l|}{ Characteristics $^{a}$} & \multirow{2}{*}{$\begin{array}{l}\text { Participants } \\
(n=470)\end{array}$} & \multirow{2}{*}{$\begin{array}{l}\text { short-response-sheet -participants }(n=107) \\
57.8(9.0)\end{array}$} & \multirow{2}{*}{$\frac{p \text {-value }}{<0.001}$} \\
\hline Age, mean (SD) & & & & \\
\hline \multirow[t]{2}{*}{ Gender, n (\%) } & Female & $160(34.0 \%)$ & $55(51.4 \%)$ & \multirow[t]{2}{*}{$<0.001$} \\
\hline & Male & $307(65.3 \%)$ & $51(47.7 \%)$ & \\
\hline \multirow[t]{4}{*}{ Employment as GP, n (\%) } & $<5$ years & $35(7.4 \%)$ & - & - \\
\hline & $5-10$ years & 95 (20.2\%) & - & - \\
\hline & $11-20$ years & $134(28.5 \%)$ & - & - \\
\hline & $>20$ years & $203(43.2 \%)$ & - & - \\
\hline \multirow[t]{3}{*}{ Location of GP's practice, n (\%) } & Urban & $119(25.3 \%)$ & - & - \\
\hline & Rural & $86(18.3 \%)$ & - & - \\
\hline & Medium-size town & $253(53.8 \%)$ & - & - \\
\hline \multicolumn{2}{|c|}{ Challenging patients, proportion (range) } & $15.96 \%(0-80 \%)$ & - & - \\
\hline
\end{tabular}

${ }^{a} \mathrm{n}$ various due to missing data; $S D$ standard deviation, GP general practitioner

personality', and 'person with a lot of questions' a strong correlation to the dependent variable 'overall job satisfaction' was found.

Table 5 shows the linear regression analysis of the individual characteristics, aspects of job satisfaction, challenging medical conditions and traits of patients which correlated significantly with the outcome variable 'overall job satisfaction'. A model with an explained variance with more than $71 \%\left(R^{2} \sim 0.714\right)$ on the outcome variable 'overall job satisfaction' was carried out. The higher overall satisfaction was associated with lower years of employment as a GP and higher satisfaction concerning nearly all aspect of job satisfaction except for recognition for work and income. The statistics of collinearity ranged between 2.406 (VIF-value), 0.416 (tolerance value) for 'employment as GP' and 1.062 (VIF-value), 0.941 (tolerance value) for 'amount of variety in job'.

Table 2 Descriptive statistics of job satisfaction $(n=470)$

\begin{tabular}{lll}
\hline Aspects of job satisfaction $^{\text {a }}$ & Mean (SD) & Cl 95\% \\
\hline Physical working condition & $4.91(1.55)$ & $4.77-5.06$ \\
Freedom of working method & $5.17(1.46)$ & $5.03-5.30$ \\
Colleagues and fellow workers & $5.80(1.26)$ & $5.68-5.92$ \\
Recognition for work & $5.44(1.40)$ & $5.31-5.57$ \\
Amount of responsibility & $5.04(1.58)$ & $4.89-5.19$ \\
Income & $4.47(1.67)$ & $4.31-4.62$ \\
Opportunity to use abilities & $5.27(1.43)$ & $5.14-5.41$ \\
Hours of work & $4.20(1.82)$ & $4.03-4.37$ \\
Amount of variety in job & $5.49(1.39)$ & $5.36-5.62$ \\
Overall job satisfaction & $5.41(1.26)$ & $5.29-5.53$ \\
\hline
\end{tabular}

arange from 1 "extreme dissatisfaction" to 7 "extreme satisfaction"; SD standard deviation, $\mathrm{Cl}$ confidence interval

\section{Discussion}

To our knowledge there has been little research on difficult medical encounters especially for their association with job satisfaction on primary care physicians in Germany. Our sample of participating GPs are comparable to the whole sample of GPs in Germany concerning age but differs slightly by gender, $34.0 \%$ women in our sample comparing to $43.9 \%$ in the whole sample of GPs in Germany [15]. Moreover, the results showed that our participants were mostly satisfied with their colleagues but dissatisfied with their income and working hours which is comparable to other studies with GPs not only in Germany [16-18].

Table 3 Descriptive statistics of challenging medical conditions of patients from the perspective of GPs

\begin{tabular}{lll}
\hline Medical Conditions $^{\mathrm{a}}$ & Mean (SD) & $\mathrm{Cl} 95 \%$ \\
\hline Somatization disorder & $7.42(2.38)$ & $7.20-7.65$ \\
Chronic pain & $7.22(2.34)$ & $7.00-7.44$ \\
Addiction to alcohol & $7.07(2.45)$ & $6.84-7.31$ \\
Schizophrenia & $6.83(2.56)$ & $6.59-7.08$ \\
Depression & $6.67(2.38)$ & $6.45-6.90$ \\
Anxiety disorder & $6.57(2.50)$ & $6.34-6.81$ \\
Dementia & $6.32(2.56)$ & $6.08-6.56$ \\
Obesity & $5.90(2.54)$ & $5.66-6.14$ \\
Polypharmacy & $5.68(2.51)$ & $5.44-5.92$ \\
Multimorbidity & $5.22(2.50)$ & $4.98-5.45$ \\
Chronic renal failure & $5.05(2.48)$ & $4.82-5.28$ \\
Diabetes type 2 & $4.87(2.50)$ & $4.64-5.11$ \\
Heart failure & $4.03(2.43)$ & $3.80-4.26$ \\
High blood pressure & $3.22(2.14)$ & $3.02-3.43$ \\
\hline
\end{tabular}

arange from 1 "not challenging at all" to 10 "very challenging"; $S D$ standard deviation, $\mathrm{Cl}$ confidence interval, $G P$ general practitioner 
Table 4 Descriptive statistics of challenging traits of patients from the perspective of GPs

\begin{tabular}{lll}
\hline Characteristic of patients $^{\mathrm{a}}$ & Mean (SD) & Cl 95\% \\
\hline Aggressive & $7.88(2.56)$ & $7.64-8.12$ \\
Demanding & $7.85(2.27)$ & $7.64-8.06$ \\
Unfriendly & $6.77(2.72)$ & $6.52-7.02$ \\
Limited compliance & $6.24(2.64)$ & $6.00-6.48$ \\
Obsessive-compulsive personality & $6.00(2.34)$ & $5.78-6.22$ \\
Person with a lot of questions & $5.68(2.53)$ & $5.44-5.91$ \\
Critical & $4.87(2.41)$ & $4.65-5.09$ \\
Anxious & $4.37(2.75)$ & $4.11-4.62$ \\
\hline
\end{tabular}

arange from 1 "not challenging at all" to 10 "very challenging"; SD standard deviation, $\mathrm{Cl}$ confidence interval, GP general practitioner

Furthermore, our results confirms findings from previous international studies dealing with challenging encounters in primary care. It could be shown that GPs perceive $16 \%$ of their patients as challenging $[1,2]$. In accordance with the statement to complex individual humans it can be assumed that both - GP and patient - meeting in a specific situation should initiated an acceptable work alliance [19]. However, for this specific situation it is a challenge, different medical conditions and traits of patients will be perceived as challenging and there is a clash of two complex systems, the system from the GP and the system from the patient. The encounter between GP and patient could be experienced as challenging from the perspective of GP and could be increased if emotional or behavioural factors from patients overlap or influence the consultation process. Moreover, this specific situation could lead to the perception a difficult encounter.

Especially 'somatization disorder', 'chronic pain' and 'addiction to alcohol' were perceived as challenging medical conditions by the participating GPs. The chronic conditions like 'high blood pressure, 'heart failure' and 'diabetes type 2' showed the lowest rate of challenging medical conditions. Consistently as challenging perceived are psychiatric diseases, whereas diseases of the cardiovascular system are considered least challenging $[4,6]$. It has been observed that 'aggressive patients' have the highest rate within the challenging traits of patients which is comparable to an interview study with family physicians. This study identified aggressive patients as the most difficult ones [20]. It can be assumed that the careful handling of challenging patients especially with psychiatric diseases could be an important part for the training to get a GP. The German training regulation for

Table 5 Associations of individual characteristics, different aspects of job satisfaction and challenging medical condition and characteristics of patients on the outcome variable overall job satisfaction (results of linear regression analysis, under specification of standardized beta coefficient, $a=5 \%)$

\begin{tabular}{|c|c|c|c|}
\hline & Variables & $\beta$ & $p$-value \\
\hline \multirow[t]{3}{*}{ Characteristic of participants } & Age & 0.097 & 0.023 \\
\hline & Gender & 0.046 & 0.128 \\
\hline & Employment as GP & -0.091 & 0.033 \\
\hline \multirow[t]{9}{*}{ Aspects of job satisfaction } & Physical working condition & 0.367 & $<0.001$ \\
\hline & Freedom of working method & 0.096 & 0.003 \\
\hline & Colleagues and fellow workers & 0.078 & 0.007 \\
\hline & Recognition for work & 0.051 & 0.078 \\
\hline & Amount of responsibility & 0.240 & $<0.001$ \\
\hline & Income & 0.052 & 0.072 \\
\hline & Opportunity to use abilities & 0.244 & $<0.001$ \\
\hline & Hours of work & 0.108 & 0.003 \\
\hline & Amount of variety in job & 0.061 & 0.033 \\
\hline \multirow[t]{4}{*}{ Challenging medical conditions of patients } & Heart failure & 0.017 & 0.675 \\
\hline & Diabetes type 2 & -0.032 & 0.399 \\
\hline & Chronic renal failure & 0.034 & 0.383 \\
\hline & Anxiety disorder & -0.035 & 0.305 \\
\hline \multirow[t]{4}{*}{ Challenging traits of patients } & Anxious & -0.008 & 0.818 \\
\hline & Unfriendly & -0.010 & 0.751 \\
\hline & Obsessive-compulsive personality & -0.031 & 0.343 \\
\hline & Person with a lot of questions & -0.034 & 0.278 \\
\hline$R^{2}$ & & 0.714 & \\
\hline
\end{tabular}


GPs ('Weiterbildungsverordnung für Ärzte') may be a cause for the challenges GPs come up with when treating patients with mental disorders, since it presumably does not stipulate the acquisition of required skills in the diagnosis and treatment of psychiatric disorders, like depression. This should have high priority due to high prevalence and therefore relevance. About $1 \%$ of German postgraduates do psychiatry rotations, thus structured training is rare [21]. In Germany, the lifetime prevalence of diagnosed depression is $15 \%$ in women and $8 \%$ in men and most of them are seen and managed in primary care [22]. In addition, depression is a costly disease not so much due to therapy costs but due to missed workdays [23].

The rotation curricula for the training of GPs in other countries, where GPs perceive psychiatric disorders as challenging as well, do not contain the acquisition of broad skills in psychiatry, neither [24]. This might explain the scientific discourse concerning the quality of care when it comes to treating patients with depression in primary care settings [25]. Thus future studies should address the questions, in what amount psychiatric competences are gained during the postgraduate training in general practises and if GPs with a mandatory rotation in psychiatry perceive these conditions as less challenging.

Surprisingly, challenging encounters described with medical conditions and traits of patients are not associated with overall job satisfaction of participating physicians. However, motivational aspects at work expressed in terms of different intrinsic and extrinsic factors are strongly associated with overall job satisfaction. It can be assumed that the feeling at the job is more relevant and generalizable than the challenging encounters for our sample. However, our results are in contrast to a study which found an interaction between lower job satisfaction and higher reporting challenging encounters [7].

\section{Strength and limitations}

This was the first cross-sectional study performed with all GPs of a complete federal state in Germany addressing challenging encounters. The study benefited from the usage of a well-known instrument which was validated in a large cohort of Australian medical practitioner [13]. The job satisfaction scale was already used in different studies about job satisfaction in primary care in Germany [16-18]. The job satisfaction scale was not specific validated for our sample. The response rate was 38\% relatively high in contrast to the statement by Kelley et al. [26]. They assumed for postal questionnaire surveys a response rate of $20 \%$ as normal for such surveys [26]. However, there is a risk of selection bias of highly motivated GPs in our results who answered to the questionnaire. Interestingly, short-response-sheet responders in our study were slightly older and more often female comparing to responders. This corresponds with findings of earlier studies that female GPs are somewhat less willing to participate in studies in primary care [27]. In addition, a potential lack of accuracy and completeness of the GP's remembrance of patients and situations might have led to recall bias and therefore a systematic error. Furthermore, the list of challenging medical conditions and traits included a high proportion of different psychiatric diseases which could influence GP's response behaviour. In addition, this was an exploratory study; $p$ values should be interpreted carefully. Moreover, there are no clear statements in the literature concerning the statistical analysis of surveys using Likert scales [28, 29]. Therefore, we handled the Likert scales as an interval which could implicated a potential statistical bias. Finally, this was a cross-sectional study, and thus, we must be cautious to derive causal links from these findings.

\section{Conclusions}

German GPs perceive 16\% of their patients as challenging. Psychiatric disorders such as somatization disorders and chronic pain are perceived as challenging. Moreover, the data shows that aggressive and demanding patients influence the perception as challenging encounter. It can be assumed that to deal with these attributes a specific training of communication skills during postgraduate training but also for experienced GPs would be necessary. The investigation in a Continuing Professional Development including communication training, conflict management and dealing with teams might be a supporting function for working as a GP and to ensure a good quality of care. Interestingly this study indicates that challenging encounter in terms of challenging medical conditions and traits of patients do not affect GP's job satisfaction. However, more research is needed concerning the connection between the perception of the complexity of challenging encounter and their impact on quality of care in longitudinal studies.

\section{Additional file}

Additional file 1: Questionnaire "Difficult medical encounters and job satisfaction". The questionnaire for the presented survey is available as additional file. (PDF $28 \mathrm{~kb}$ )

\section{Abbreviations}

BBSR: Federal Institute for Research on Building, Urban Affairs and Spatial Development; Cl: Confidence interval; GP: General practitioner; SD: Standard deviation; SPSS: Statistical package of social science; STROBE-

Guidelines: Strengthening the Reporting of Observational Studies in Epidemiology; VIF: variance inflation factor 


\section{Funding}

This research received no specific grant from any funding agency in the public, commercial or not-for-profit sectors.

\section{Availability of data and materials}

The datasets used and during the current study are available from the corresponding author on reasonable request.

\section{Authors' contributions}

All authors contributed to this study. JS was the project manager for the development of the questionnaire. AK, JM and KG made substantial contributions to conception and design of date. AK and JM were responsible for acquisition of data. KG performed the statistical analyses and drafted the manuscript. AK, JM and JS critically revised the manuscript draft and approved its final version. All authors have read and approved the final version of this manuscript and agreed to be accountable for all aspects of the work.

\section{Ethics approval and consent to participate}

Ethical approval for this research study was obtained from the University of Luebeck in May 2015 (Approval No. 15-110). No additional data were evaluated. The return of the anonymous paper-based questionnaire was classified as informed consent.

\section{Competing interests}

The author KG is an associate editor for BMC Family Practice. The others authors declare that they do not have any competing interests.

\section{Publisher's Note}

Springer Nature remains neutral with regard to jurisdictional claims in published maps and institutional affiliations.

Received: 27 November 2017 Accepted: 23 April 2018

\section{Published online: 09 May 2018}

\section{References}

1. Hahn SR, Kroenke K, Spitzer RL, Brody D, Williams JB, Linzer M. The difficult patient: prevalence, psychopathology, and functional impairment. J Gen Intern Med. 1996;11:1-8.

2. Hinchey SA, Jackson JL. A cohort study assessing difficult patient encounters in a walk-in primary care clinic, predictors and outcomes. J Gen Intern Med. 2011;26:588-94.

3. Cannarella Lorenzetti R, Jaques M, Donovan C, Cottrell S, Buck J. Managing difficult encounters: understanding physician, patient, and situational factors. Am Fam Physician. 2013:87:419-25.

4. Haas $L$, Leiser JP, Magill MK, Sanyer ON. Management of the difficult patient. Am Fam Physician. 2005;72:2063-8.

5. DeMarco MA, Nogueira-Martins LA, Yazigi L. Difficult patients or difficult encounters. QJM. 2005:98:542-3.

6. Arndt B, Tuan WJ, White J, Schumacher J. Panel workload assessment in US primary care: accounting for non-face-to-face panel management activities. J Am Board Fam Med. 2014;27:530-7.

7. An PG, Rabatin JS, Manwell LB, Linzer M, Brown RL, Schwartz MD. Burden of difficult encounters in primary care: data from the minimizing error, maximizing outcomes study. Arch Intern Med. 2009;169:410-4.

8. An PG, Manwell LB, Williams ES, Laiteerapong N, Brown RL, Rabatin JS, et al. Does a higher frequency of difficult patient encounters lead to lower quality care? J Fam Pract. 2013;62:24-9.

9. Wallace JE, Lemaire JB, Ghali WA. Physician wellness: a missing quality indicator. Lancet. 2009:374:1714-21.

10. Von Elm E, Altman DG, Egger M, Pocock SJ, Gotzsche PC, Vandenbroucke $J P$. The strengthening the reporting of observational studies in epidemiology (STROBE) statement: guidelines for reporting observational studies. Ann Intern Med. 2007:147:573-7.

11. Federal Institute for Research on Building, Urban Affairs and Spatial Development. http://www.bbsr.bund.de/BBSR/DE/Raumbeobachtung/ Downloads/downloads_node.htm|\#doc443480bodyText1. Accessed 10 July 2017.

12. Warr PJ, Cook J, Wall T. Scales for the measurement of some work attitudes and aspects of psychological well-being. J Occup Psychol. 1979;52:129-48.

13. Hills $D$, Joyce $C$, Humphreys J. Validation of a job satisfaction scale in the Australian clinical medical workforce. Eval Health Prof. 2012;35:47-76.
14. Field A. Discovering statistics using SPSS. London: Sage Publications Ltd; 2011.

15. Database of the Association of Statutory Health Insurance Physicians. http://gesundheitsdaten.kbv.de/cms/html/16392.php. Accessed 20 Nov 2017.

16. Goetz K, Campbell SM, Steinhaeuser J, Broge B, Willms S, Szecsenyi J. Evaluation of job satisfaction of practice staff and general practitioners: an exploratory study. BMC Fam Pract. 2012;12:137.

17. Goetz K, Jossen M, Szecsenyi J, Rosemann T, Hahn K, Hess S. Job satisfaction of primary care physicians in Switzerland: an observational study. Fam Pract. 2016:33:498-503.

18. Goetz K, Broge B, Willms S, Joos S, Szecsenyi J. Die Arbeitszufriedenheit von Allgemeinmedizinern. Med Klinik. 2010;105:767-71.

19. Miles A. Complexity in medicine and healthcare: people and systems, theory and practice. J Eval Clinic Pract. 2009;15:409-10.

20. Steinmetz D, Tabenkin H. The 'difficult patient' as perceived by family physicians. Fam Pract. 2001;18:495-500.

21. Förderung der Weiterbildung in der Allgemeinmedizin in der ambulanten und stationären Versorgung Evaluationsbericht für das Jahr 2014. http:// www.kbv.de/media/sp/2015_11_26_Evaluationsbericht_Foerderung_ Weiterbildung_2014_fin.pdf. Accessed 10 July 2017.

22. Busch MA, Maske UE, Ryl L, Schlack R, Hapke U. Prävalenz von depressiver Symptomatik und diagnostizierter Depression bei Erwachsenen in Deutschland. Bundesgesundheitsbl. 2013;56:733-9.

23. Luppa M, Heinrich S, Angermeyer MC, König HH, Riedel-Heller SG. Cost-of-illness studies of depression: a systematic review. J Affect Disord. 2007;98:29-43.

24. ACGME Program Requirements for Graduate Medical Education in Family Medicine. http://www.acgme.org/Portals/0/PFAssets/ProgramRequirements/ 120_family_medicine_2017-07-01.pdf?ver=2017-06-30-083354-350. Accessed 10 July 2017.

25. Schumann I, Schneider A, Kantert C, Löwe B, Linde K. Physicians' attitudes, diagnostic process and barriers regarding depression diagnosis in primary care: a systematic review of qualitative studies. Fam Pract. 2012;29:255-63.

26. Kelley K, Clark B, Brown V, Sitzia J. Good practice in the conduct and reporting of survey research. Int I Qual Health Care. 2003;15:261-6.

27. Peters-Klimm F, Hermann K, Gágyor I, Haasenritter J, Bleidorn J. Experiences and attitudes regarding practice-based clinical trials: results of a survey among German primary care physicians. Gesundheitswesen. 2013;75:321-7.

28. Norman G. Likert scales, levels of measurement and the "laws" of statistics. Adv Health Sci Educ Theory Pract. 2010;15:625-32.

29. Carifio J, Perla R. Resolving the 50-year debate around using and misusing Likert scales. Med Educ. 2008:42:1150-2.

\section{Ready to submit your research? Choose BMC and benefit from:}

- fast, convenient online submission

- thorough peer review by experienced researchers in your field

- rapid publication on acceptance

- support for research data, including large and complex data types

- gold Open Access which fosters wider collaboration and increased citations

- maximum visibility for your research: over $100 \mathrm{M}$ website views per year

At BMC, research is always in progress.

Learn more biomedcentral.com/submissions 